\title{
Papers
}

\section{MRSA bacteraemia in patients on arrival in hospital: a cohort study in Oxfordshire 1997-2003}

\author{
David H Wyllie, Tim E A Peto, Derrick Crook
}

\begin{abstract}
Objective To describe the incidence and determinants of methicillin resistant and methicillin sensitive Staphylococcus aureus (MRSA and MSSA) bacteraemia in patients presenting to acute hospitals.

Design Anonymised record linkage study with information from hospital information systems and microbiology databases. Setting One teaching hospital and one district general hospital in Oxfordshire.

Participants All patients admitted to a teaching hospital 1 April 1997 to 31 March 2003 and to a district general hospital 1 April 1999 to 31 March 2003.

Main outcome measures Detection of MRSA and MSSA from blood cultures taken during the first two days of admission to hospital.

Results In the teaching hospital, there were 479 patients with MSSA and 116 with MRSA bacteraemia admitted from the community. Among this group, which comprised $24 \%$ of all hospital MRSA cases, 31\% (36 cases) of patients had been admitted to renal, oncology, or haematology services for intensive day case therapy. The $69 \%$ remaining were most commonly patients admitted as medical or surgical emergencies. At least $91 \%$ had been in hospital previously; the median time since discharge was 46 days. About half of cases were in patients in whom MRSA had not been isolated before. Similar epidemiology was observed in the district general hospital.

Conclusion Diagnostic algorithms and policies on use of antibiotics need to reflect the fact that a quarter of hospital MRSA cases occur in patients who have previously been in hospital and are subsequently readmitted.
\end{abstract}

\section{Introduction}

Highly successful clones of methicillin resistant Staphylococcus aureus (MRSA) established themselves in UK hospitals in the 1990s. ${ }^{1}$ Although other MRSA clones, which have a different genetic makeup, have spread in communities in the United States and elsewhere, ${ }^{2}$ in the United Kingdom MRSA is still thought to be predominantly hospital based. ${ }^{3}$ The national surveillance scheme for infection of the blood stream with MRSA counts MRSA bacteraemia by trust ${ }^{4}$ but does not yet contain the detail included in earlier schemes. ${ }^{5}$ In particular, so far it has not addressed whether MRSA bacteraemia is being imported into hospitals from the community. The Department of Health requires reductions in MRSA bacteraemia rates in hospital by $60 \%{ }^{6}$ To decide where to target interventions for infection control, we surveyed the epidemiology of MRSA and methicillin sensitive $S$ aureus (MSSA) bacteraemia in two Oxfordshire hospitals over a seven year period.

\section{Methods}

\section{Data sources and study design}

Our study involved the John Radcliffe, Radcliffe Infirmary, and Churchill Hospitals, which operate together as one acute care teaching hospital in Oxford, also offering specialist regional services including cardiothoracic and neurosurgery, and nephrology. The Horton Hospital is a district general hospital in Banbury, 30 miles $(48 \mathrm{~km})$ further north. Together, they provide all acute clinical and bacteriology services to a catchment area of about 600000 people.

Our first study was done in the seven years to 31 March 2004 in the Oxford hospitals. To confirm our observations from the Oxford site we performed a second study over the five years to 31 March 2004 in the Banbury hospital. These timings were chosen to coincide with financial years. Patient administration system (PAS) data were available for three months before the start of the study in both hospitals. We were unable to study the Horton Hospital for exactly the same period as the Oxford hospitals because of differences in administration systems between the two sites before 1999. Figure 1 shows the durations of the study and the sources of data.

\section{Anonymisation}

Data were anonymised with routines written in Visual FoxPro 8 (Microsoft) with the Crypt 2 ActiveX plug-in (Chilkat Software, http://www.chilkatsoft.com), by transforming hospital number, NHS number, and name and date of birth into code numbers with a salted MD5 hash function, which is an irreversible step. To allow calculation of age, we recorded the month of birth of each case.

\section{Record linkage}

We devised and tested a method of linking anonymised hospital microbiology results, general practice microbiology results, and patient activity data, based on clustering results sharing hospital numbers, NHS numbers, and combinations of surname, first initial, and date of birth. All hospital activity data from the patient administration system could be linked, but a small number of microbiology samples could not. Of the 802036 specimens from which $S$ aureus could be cultured, we excluded $5864(0.7 \%)$ because they lacked any adequate identifiers to make links. Among the 96463 blood cultures analysed, we excluded 105 $(0.1 \%)$ because of inadequate identifiers.

\section{Specialties of cases}

We used nationally recognised admission specialty codes to group cases. The patient administration system records each epi- 
sode of admission to hospital, including admissions for haemodialysis and day care, as well as longer admissions. To simplify analysis, we grouped some specialties, based on similar activity, as follows: surgical specialties except trauma (codes 100$110,150,160,170,171)$; otorhinolaryngology, eye, and oral surgery $(120,130,140)$; haematology and oncology $(303,370,800)$; medical specialties $(300,301,302,330,340,350)$; paediatrics (400-420), and obstetrics and gynaecology $(500,501,502,560$, 610). Acute medicine accepts patients of all ages. We differentiated between elective admissions, emergency transfers, and transfers between hospitals to subdivide cases for some analyses.

\section{Screening policies}

During the study period, our MRSA screening policies were based on national guidance ${ }^{7}$; screening for MRSA on admission was recommended on high risk units (renal, intensive care, and some surgical wards).

\section{Microbiological processing}

Bacteraemia was detected in Bactec F+ blood culture bottles, incubated for five days or for 21 days if endocarditis was suspected. Identification of $S$ aureus was with tube coagulase and DNAse tests, and sensitivity testing for methicillin was performed by disc diffusion with methicillin or oxacillin discs and Colombia agar with $2 \%$ salt. $^{8}$

\section{Case definitions}

As recommended by the UK mandatory surveillance scheme ${ }^{4}$ we defined a "case of $S$ aureus bacteraemia" as isolation of $S$ aureus from blood cultures, unless $S$ aureus had been isolated from blood cultures within the two previous weeks. "Admission $S$ aureus bacteraemia" was defined as those cases of $S$ aureus bacteraemia detected in the first two days after admission to the hospital $^{9}$ unless the patient had been transferred from another hospital.

\section{Definition of previous isolation of MRSA and previous hospital stay}

We classified patients as having "previous MRSA isolation" when there was a record of MRSA being isolated from any samples at any time between 1 January 1995 and the date of admission. "Previous admission" was defined as having a record of a previous hospital admission between 1 January 1997 and the date of the current admission. Thus, at the start of the study on 1 April 1997, three months' follow-up was available. "Previous hospital stay" was calculated as the total time, in minutes, for which the patient had been in hospital before the current admission. A stay of 24 hours was counted as one day-for example, six sessions of haemodialysis lasting four hours each counted as one day, as did 24 hours as an inpatient.

\section{Statistical analysis}

We used SPSS version 12 for Windows for all analyses. We used a $\chi^{2}$ test to examine whether MRSA and MSSA isolates occurred in similar proportions in admission samples compared with later samples obtained after admission. To investigate whether MRSA was becoming more common in admission $S$ aureus bacteraemia isolates over time, we used conventional logistic regression analysis to generate a maximal likelihood estimate (MLE) of $\mathrm{B}$ where $\operatorname{Logit}(p)=B t+c$, where $c$ is a constant, $p=1$ for cases of MRSA and 0 for cases of MSSA, and t is the time in years since 1 January 1995. Examining all isolates to the Oxford hospitals, we used a similar approach to determine whether previous MRSA isolation was becoming more common among admissions over time. We report the MLE of $\mathrm{e}^{\mathrm{B}}$ and its 95\% confidence intervals.

We analysed the Oxford cohort of patients in two separate groups to examine the relation between MRSA or MSSA isolation and the ages and previous hospital exposures of patients. These groups were cases in patients admitted to renal, oncology or haematology wards, and all other cases. We performed one way analysis of variance between groups with different blood culture results. Where there was evidence of heterogeneity between groups, as judged by the significance of $F$, we performed post hoc multiple comparisons between groups, calculating their significance using Schleffe's method with the SPSS ONEWAY command.

Durations of stay were compared with Mann-Whitney U tests. We calculated the interval between the admission of interest and any discharge that had occurred within the 500 preceding days. We compared median times since last discharge using Mann-Whitney U tests, assigning a value of 501 days to those patients with no record of admission within 500 days.

\section{Results}

Increase in MRSA bacteraemia presenting on arrival

During the period of study there were 974 cases of MSSA and 493 cases of MRSA bacteraemia in the Oxford teaching hospitals. There were 697 cases of $S$ aureus bacteraemia within the first two days after arrival. Of these, 102 cases were in patients who had just been transferred from another hospital; in the rest of our analysis, we focused only on the 595 patients admitted from the community (table 1). Admission cultures accounted for $49 \%(479 / 974)$ of total hospital MSSA and 24\% (116/493) of total hospital MRSA bacteraemia episodes (proportions significantly different, $\left.\chi^{2}=89, \mathrm{P}<0.001\right)$. Both MRSA and MSSA

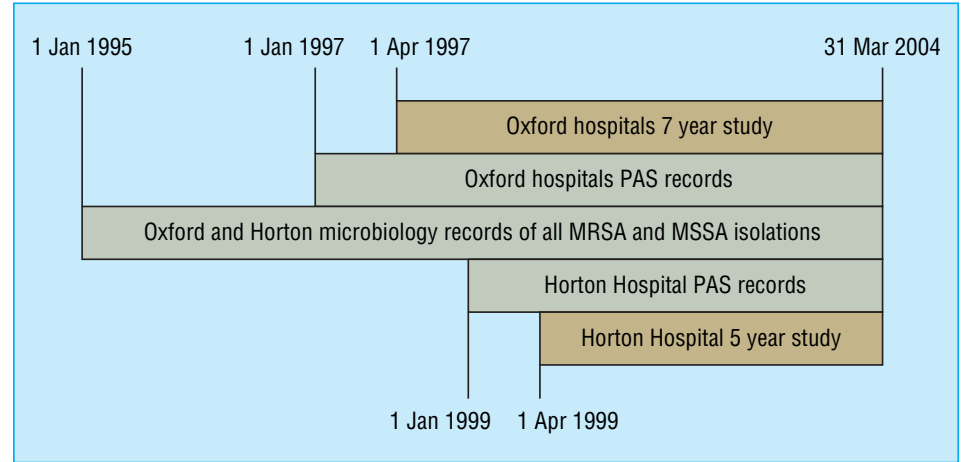

Fig 1 Duration of studies and sources of data. The two studies used linked microbiology and patient administration system (PAS) data. Study periods and durations for which data were available are shown 


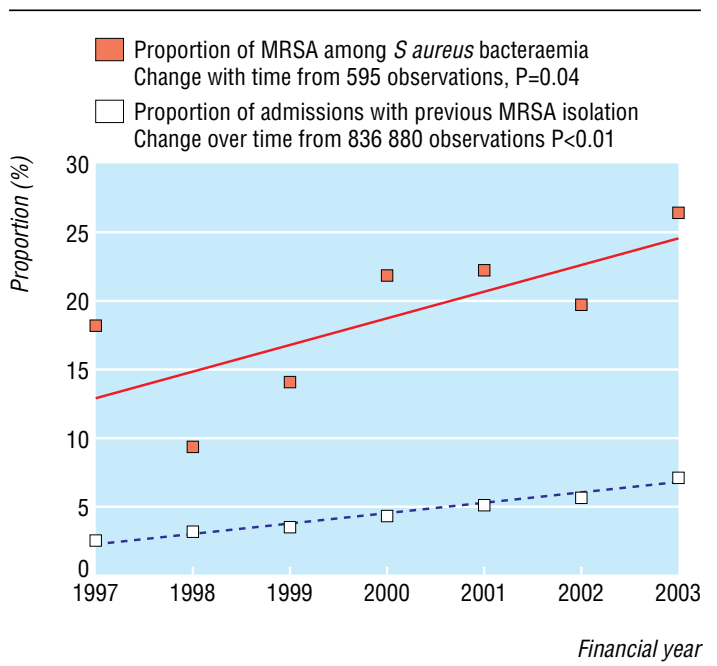

Fig 2 Increase in MRSA over time, showing proportion of methicillin resistant isolates among admission $S$ aureus isolates in Oxford hospitals and proportion of all admissions with previous MRSA isolation, year by year

were recovered in three cases, which we excluded from further analysis. Notably, the proportion of methicillin resistance among admission $S$ aureus bacteraemias rose significantly from $14 \%$ (16/115) in 1997 and 1998 to 26\% (25/105) in 2003 (increase per year $1.11,95 \%$ confidence interval 1.01 to 1.24 ). This period also saw marked increases in MRSA isolation from sites other than blood and a highly significant increase in the proportion of patients admitted with previous MRSA isolation from 2.6\% to $7.2 \%$ between 1997 and 2003 (increased per year 1.19, 1.18 to 1.20 , fig 2).

\section{Previous MRSA isolation and admission $S$ aureus bacteraemia}

Previous MRSA isolation from any site was strongly associated with admission MRSA compared with MSSA bacteraemia (fig 3). Despite this, among patients with admission MRSA bacteraemia about half $(56 / 116,48 \%)$ had no record of previous MRSA isolation.

\section{Epidemiology of admission $S$ aureus bacteraemia}

Admission $S$ aureus bacteraemia occurred predominantly in patients who had previously been admitted to hospital: of all admissions, $91 \%$ of patients with MRSA bacteraemia (106/116) and $77 \%$ of cases with MSSA (369/479) had previous hospital contact recorded. At the start of the study, we had only three months' information of previous hospital attendance. Compatible with an underestimation of previous hospital exposure, the patients with admission MRSA bacteraemia and no record of previous admission were over-represented the start of the study, with four identified in 1997, two in 1998, and one each in 1999, 2000, 2001, and 2003. Two of them lived outside our normal catchment area. These patients were of similar age (means $74.3 \mathrm{v}$ $67.3,95 \%$ confidence interval for difference -1.34 to 15.3 ) to those with previous contact.

Table 1 shows current admission and previous discharge specialties. The rate of current admission $S$ aureus bacteraemia among patients without previous admission was 40/100 000; rates among previously well neonates and those discharged from specialities such as otorhinolaryngology, oral surgery, and ophthalmology were similar. By contrast, rates of re-admission $S$ aureus bacteraemia after discharge from other specialties such as emergency medicine and trauma were fivefold higher at over 200/100 000 discharges.

We analysed the time since last discharge and the total duration of stay before admission in more detail, separating renal, haematology and oncology cases from the others, a distinction made because of the regular day case care of patients attending such services. The epidemiology of these two groups is different (fig 3; table 2 shows means and significance tests). Among the patients admitted to these services, $90 \%$, with or without any sort of bacteraemia, had visited hospital within the past 16 days. MRSA isolation was unrelated to previous time in hospital, and the age of patients with MRSA did not differ from the age of those without bacteraemia (fig 4, table 2). For admissions to other specialties, isolation of MRSA was associated with older patients and with longer hospital stay (table 2). In patients with MRSA the mean time since previous discharge was 46 days compared with 104 days in patients with MSSA and patients without

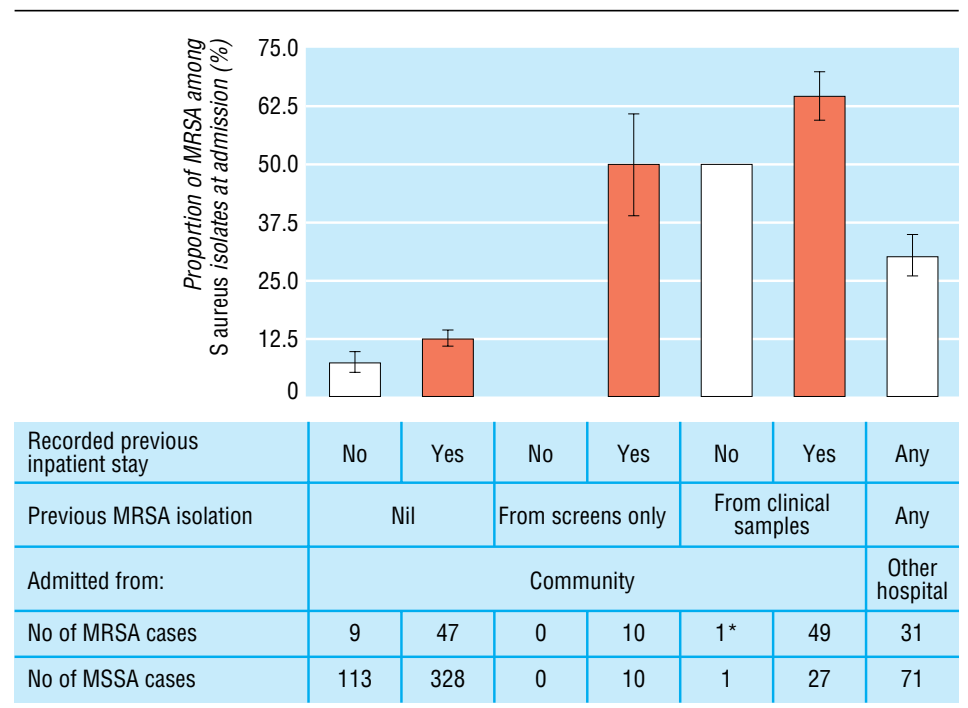

Fig 3 Association between MRSA bacteraemia and previous MRSA isolation and previous hospital stay, showing previous hospital stay, previous MRSA isolation, and numbers of $S$ aureus isolates and proportions of MRSA among isolates obtained in Oxford hospitals. *This patient had previously had MRSA isolated from swab taken by general practitioner 
Table 1 Patients with admission $S$ aureus bacteraemia according to specialty of current admission and specialty they were discharged from on previous admission

\begin{tabular}{|c|c|c|c|c|c|c|c|c|c|c|c|c|c|c|c|c|}
\hline & \multicolumn{8}{|c|}{ Current admission from community } & \multicolumn{8}{|c|}{ Previous discharge } \\
\hline & \multicolumn{4}{|c|}{ Admission bacteraemia } & \multicolumn{3}{|c|}{ Rate/100 000 admissions } & \multirow{2}{*}{$\begin{array}{c}\text { Proportion } \\
\text { of MRSA } \\
(\%)\end{array}$} & \multicolumn{4}{|c|}{ Admission bacteraemia } & \multicolumn{3}{|c|}{ Rate/100 000 admissions } & \multirow{2}{*}{$\begin{array}{c}\text { Proportion } \\
\text { of MRSA } \\
(\%)\end{array}$} \\
\hline & No & MRSA & MSSA & Both & $\begin{array}{c}\text { Any } \\
\text { MRSA }\end{array}$ & Any MSSA & $\begin{array}{l}\text { Any } S \\
\text { aureus }\end{array}$ & & No & MRSA & MSSA & Both & $\begin{array}{c}\text { Any } \\
\text { MRSA }\end{array}$ & $\begin{array}{c}\text { Any } \\
\text { MSSA }\end{array}$ & $\begin{array}{l}\text { Any } S \\
\text { aureus }\end{array}$ & \\
\hline Trauma & 17845 & 2 & 6 & 0 & 11 & 34 & 25 & 25 & 5918 & 6 & 7 & 0 & 101 & 118 & 220 & 46 \\
\hline ENT, eye, oral & 64471 & 1 & 1 & 0 & 2 & 2 & 3 & 50 & 28125 & 2 & 12 & 0 & 7 & 43 & 50 & 14 \\
\hline $\begin{array}{l}\text { Haematology, } \\
\text { oncology }\end{array}$ & 58463 & 7 & 38 & 0 & 12 & 65 & 77 & 15 & 52170 & 6 & 36 & 0 & 11 & 69 & 80 & 14 \\
\hline Cardiology & 20125 & 0 & 3 & 0 & 0 & 15 & 15 & 0 & 10471 & 2 & 11 & 1 & 19 & 105 & 134 & 15 \\
\hline Nephrology & 192795 & 29 & 148 & 2 & 15 & 77 & 93 & 16 & 190746 & 28 & 145 & 1 & 15 & 76 & 91 & 16 \\
\hline Healthy neonates & 47833 & 0 & 1 & 0 & 0 & 2 & 2 & 0 & 9092 & 0 & 4 & 0 & 0 & 44 & 44 & 0 \\
\hline $\begin{array}{l}\text { Obstetrics, } \\
\text { gynaecology }\end{array}$ & 128540 & 0 & 2 & 0 & 0 & 2 & 2 & 0 & 74978 & 0 & 4 & 0 & 0 & 5 & 5 & 0 \\
\hline Other & 10974 & 0 & 3 & 0 & 0 & 27 & 27 & 0 & 4798 & 1 & 7 & 0 & 21 & 146 & 167 & 13 \\
\hline Elective surgery & 89997 & 0 & 1 & 0 & 0 & 1 & 1 & 0 & 42345 & 10 & 24 & 0 & 24 & 57 & 80 & 29 \\
\hline Emergency surgery & 34043 & 16 & 28 & 0 & 47 & 82 & 129 & 36 & 20861 & 10 & 19 & 0 & 47 & 91 & 139 & 34 \\
\hline Elective medicine & 43546 & 5 & 3 & 0 & 11 & 7 & 18 & 63 & 25367 & 3 & 7 & 0 & 12 & 27 & 39 & 30 \\
\hline Elective paediatrics & 20037 & 0 & 2 & 0 & 0 & 10 & 10 & 0 & 13777 & 0 & 11 & 1 & 0 & 80 & 87 & 0 \\
\hline Emergency medicine & 80693 & 55 & 214 & 0 & 68 & 264 & 333 & 0 & 45337 & 37 & 74 & 0 & 81 & 163 & 245 & 33 \\
\hline $\begin{array}{l}\text { Emergency } \\
\text { paediatrics }\end{array}$ & 21746 & 0 & 29 & 1 & 0 & 133 & 138 & 20 & 10052 & 1 & 8 & 0 & 10 & 80 & 90 & 11 \\
\hline $\begin{array}{l}\text { Palliative and pain } \\
\text { care }\end{array}$ & 5174 & 1 & 0 & 0 & 19 & 0 & 19 & 1 & 2651 & 0 & 0 & 0 & 0 & 0 & 0 & 0 \\
\hline $\begin{array}{l}\text { No recorded previous } \\
\text { admission }\end{array}$ & - & - & - & - & - & - & - & - & 299594 & 10 & 110 & 0 & 3 & 37 & 40 & 8 \\
\hline Total of above & 836282 & 116 & 479 & 3 & 17 & 63 & 80 & 21 & 836282 & 116 & 497 & 3 & 14 & 57 & 71 & 19 \\
\hline $\begin{array}{l}\text { Discharged } \geq 90 \\
\text { days }\end{array}$ & - & - & - & - & - & - & - & - & 680826 & 92 & 376 & 2 & 14 & 55 & 69 & 20 \\
\hline $\begin{array}{l}\text { Discharged }<90 \\
\text { days }\end{array}$ & - & - & - & - & - & - & - & - & 155456 & 24 & 103 & 1 & 15 & 66 & 82 & 19 \\
\hline $\begin{array}{l}\text { Additional cases } \\
\text { admitted from } \\
\text { other hospitals }\end{array}$ & - & - & - & - & - & - & - & - & 36249 & 31 & 71 & 1 & 85 & 195 & 284 & 30 \\
\hline
\end{tabular}

ENT=otorhinolaryngology.

bacteraemia $(\mathrm{P}<0.001$ for difference in MRSA $v$ MSSA and MRSA $v$ no bacteraemia, Mann-Whitney U tests) (fig 4).

\section{Similar epidemiology in a district general hospital}

To confirm whether our findings might apply elsewhere, we studied records from a district general hospital, which lacks specialist acute renal and haematology services. Among the 54749 admissions from the community during April 1999 to March 2004, there were 46 cases of admission $S$ aureus bacteraemia, of which 37 were MSSA and $9(20 \%)$ were MRSA. The nine cases of MRSA represented $27 \%$ of the 33 cases of MRSA in the hospital during that time. Only one of the nine patients had previously been in the Oxford hospitals. The patients with admission MRSA bacteraemia were significantly older than those with MSSA (75.9 $v 61.5$ years, $\mathrm{P}=0.01)$ and had spent longer in hospital $(8.1 v 1.5$ weeks, $\mathrm{P}<0.01)$. The proportion of methicillin resistance among admission $S$ aureus increased over time (increase per year 3.7, 1.5 to 9.2). Therefore, the epidemiology of admission MRSA bacteraemia in the district general hospital was similar to that in the Oxford teaching hospitals.

\section{Discussion}

In this cohort study we found that patients with MRSA bacteraemia tended to be older and were more likely to have been admitted to hospital previously. Ensuring that patients presenting with MRSA bacteraemia are recognised and receive appropriate therapy is essential, ${ }^{10}$ given the high mortality associated with the disease. As most empirical treatment for community acquired sepsis is not active against MRSA, our finding that admission MRSA bacteraemia is increasing and is responsible for about a quarter of total hospital MRSA bacteraemia in two different hos-

Table 2 Characteristics of patients admitted from community with $S$ aureus bacteraemia in Oxford hospitals, 1997-2003, according to specialty

\begin{tabular}{|c|c|c|c|c|c|c|c|c|c|}
\hline & \multirow[b]{2}{*}{ None } & \multirow[b]{2}{*}{ MRSA } & \multirow[b]{2}{*}{ MSSA } & \multirow[b]{2}{*}{ Both } & \multirow[b]{2}{*}{$F(2 \mathrm{df})$} & \multirow[b]{2}{*}{$P$ value } & \multicolumn{3}{|c|}{ Difference in means $(95 \% \mathrm{Cl})$} \\
\hline & & & & & & & MRSA $v$ MSSA & None $v$ MSSA & None $v$ MRSA \\
\hline \multicolumn{10}{|c|}{ Renal/haematology/oncology } \\
\hline No of cases & 251258 & 36 & 186 & 2 & - & - & - & - & - \\
\hline Mean age (years) & 61.8 & 60.6 & 52.9 & 88 & 24 & $<0.01$ & $7.7(-0.04$ to 5.4$)$ & 8.8 (5.7 to 12.0) & $1.2(-5.8$ to 8.2$)$ \\
\hline $\begin{array}{l}\text { Previous duration in } \\
\text { hospital (weeks) }\end{array}$ & 7.88 & 7.69 & 6.93 & 3.5 & 1.3 & 0.27 & - & - & - \\
\hline \multicolumn{10}{|l|}{ Other specialties } \\
\hline No of cases & 585024 & 80 & 293 & 1 & - & - & - & - & - \\
\hline Mean age (years) & 40.6 & 71.4 & 55.2 & 16 & 85 & $<0.01$ & 16.1 (7.3 to 25.0$)$ & 14.6 (10.6 to 18.6) & 30.7 (22.9 to 38.7) \\
\hline $\begin{array}{l}\text { Previous duration in } \\
\text { hospital (weeks) }\end{array}$ & 0.89 & 6.86 & 2.44 & 11.1 & 183 & $<0.01$ & 4.4 (3.5 to 5.4$)$ & 1.5 (1.1 to 2.0$)$ & $6.0(5.1$ to 6.8$)$ \\
\hline
\end{tabular}


pital settings should prompt review of the clinical approach to at-risk populations.

\section{Groups at risk of admission MRSA bacteraemia}

We identified two discrete groups at risk of admission MRSA bacteraemia. The first comprised patients regularly attending for renal, oncology, or haematology care, a group known to have a high use of intravascular devices. The second group, which formed about $70 \%$ of cases in our hospitals, is more difficult to define clinically. They are predominantly elderly, have had extensive previous contact with health care, and may, if US studies can be extrapolated to our population, have risk factors for diabetes, ulcers, or urinary catheters. ${ }^{11-13}$ Only about half the patients with MRSA bacteraemia on admission were known to have had MRSA before.

The limitations of our passive surveillance mean that we cannot exclude small numbers of true community acquired cases of MRSA bacteraemia; however, our surveillance does not suggest that people who have never been in hospital before make a substantial contribution to the numbers of patients with admission MRSA bacteraemia. Thus, we believe that acquisition associated with health care remains critical for MRSA bacteraemia on arrival.

\section{Clinical implications}

Detection of bacteraemia by clinical examination is difficult in elderly patients. ${ }^{14}$ Laboratory measures of host inflammatory response, such as neutrophilia, lymphopenia, and $\mathrm{C}$ reactive protein, are often of limited use, ${ }^{15}$ and procalcitonin seems to have value only when used as a rule out test. ${ }^{16}{ }^{17}$ Until at-risk groups can be more specifically identified, we suggest that in hospitals with MRSA epidemiology similar to ours empirical anti-MRSA treatment should be considered for patients with sepsis who have previously been admitted. The choice of agents is problematic, however, as widespread use of aminoglycosides, linezolid, or glycopeptides pending results of blood culture is likely to contribute to cost, spread of resistance, and drug toxicity in an already vulnerable population.

\section{Limitations of the study}

Our study has limitations. In our seven year survey, only $9 \%$ of cases had no recorded hospital contact. However, we do not have information on exposure to nursing and residential homes, contact with hospitals outside Oxfordshire or before 1997, or recent migration into the catchment area, factors that may explain the absence of recorded contact in the $9 \%$. The similarity of age between patients with MRSA with and without recorded hospital exposure supports this explanation, as opposed to the true community acquired disease seen in other countries. ${ }^{2}$ This limitation does not alter our conclusion that, of the cases of MRSA bacteraemia detected in hospital, a quarter occur in patients who have just arrived from the community, that this proportion is increasing, and that admission MRSA bacteraemia remains strongly associated with previous hospital contact.

\section{Previous MRSA isolation and admission MRSA bacteraemia}

This study reports an increased risk of admission MRSA bacteraemia in patients with previous MRSA isolation, something compatible with smaller prospective ${ }^{18}$ and retrospective ${ }^{19}$ studies showing increased MRSA disease in MRSA carriers in hospital. As recommended by UK national guidelines, ${ }^{7}$ we screened for MRSA on arrival only in selected units-including intensive care, renal and vascular surgery, and trauma wards-an approach that fails to detect some MRSA positive patients. ${ }^{20}{ }^{21}$ While a screening policy concentrated on specialties with patients at high risk of bacteraemia may have overestimated the real risk associated with MRSA carriage, our results are relevant to other centres operating similar screening policies. ${ }^{7}$

\section{Bacteraemia associated with health care}

In the US, the association between previous health care and admission bacteraemia with resistant organisms ${ }^{22}{ }^{23}$ has led to

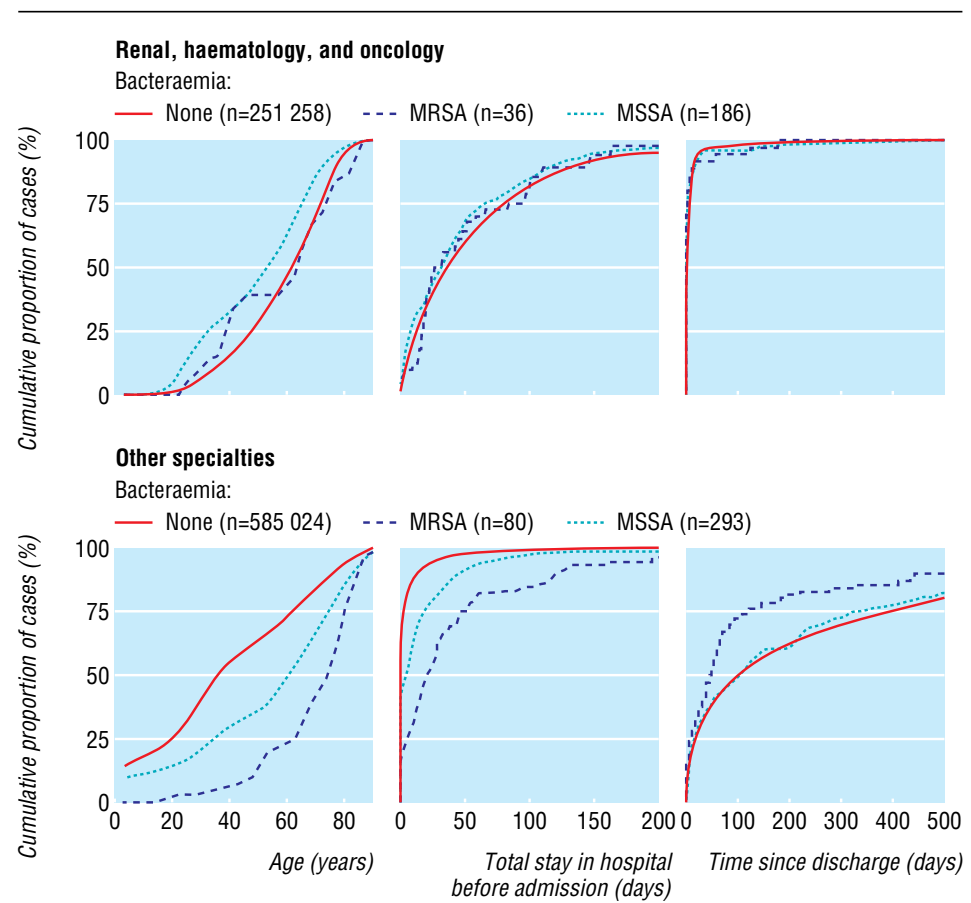

Fig 4 Timing of hospital discharge relative to admission bacteraemia: interval between last hospital admission and current admission among admissions to 0xford hospitals for renal, haematology, and oncology patients and for all other cases. Each line on graph represents proportion of patients with particular blood culture result who had been admitted within particular time 


\section{What is already known on this topic}

In the past 10 years MRSA bacteraemia has increased in the United Kingdom and affected patients are being admitted to hospital from the community

In other countries, MRSA is spreading in patients who have never been in hospital

\section{What this study adds}

Affected patients admitted from the community accounted for $25 \%$ of total hospital MRSA bacteraemia in a seven year cohort study and numbers are growing over time

Most patients ( $\geq 90 \%$ ) had previously been in hospital, half had never had MRSA isolated before, and $70 \%$ were admitted to emergency medical and surgical services

proposals to refine surveillance schemes using a new category of healthcare associated bacteraemia. ${ }^{24}$ Our data suggest healthcare associated bacteraemia presenting on arrival is an important contributor to total UK rates of hospital MRSA. The UK mandatory MRSA surveillance scheme ${ }^{4}$ is being extended and will soon be able to determine the prevalence of this problem nationally (G Duckworth, personal communication). Additional research should be undertaken to define the best way to define and manage these patients because, irrespective of intensification measures to control hospital based infection, ${ }^{6}$ in the short term ongoing presentation of MRSA bacteraemia to acute services is likely given the longevity of MRSA carriage ${ }^{19}$ and the substantial pool of patients positive MRSA in the community.

We thank Dr I Bowler for helpful comments.

Contributors: DHW obtained and linked the data, wrote the paper, and is guarantor. DHW and TEAP analysed the data. All authors contributed to the paper.

Funding: None,

Competing interests: None declared.

Ethics approval: Not required.

1 Speller DC, Johnson AP, James D, Marples RR, Charlett A, George RC. Resistance to methicillin and other antibiotics in isolates of Staphylococcus aureus from blood an cerebrospinal fluid, England and Wales, 1989-95. Lancet 1997;350:323-5.

2 Fridkin S, JC H. Methicillin-resistant S aureus disease in three communities. $N$ Engl Med 2005;352:1436-44

3 Maudsley J, Stone SP, Kibbler CC, Iliffe SR, Conaty SJ, Cookson BD, et al. The community prevalence of methicillin-resistant Staphylococcus aureus (MRSA) in older people living in their own homes: implications for treatment, screening and surveillance in the UK. J Hosp Infect 2004;57:258-62.

4 Health protection Agency Communicable Disease Surveillance Centre for the Department of Health. MRSA surveillance system-results. www.dh.gov.uk/ PublicationsAndStatistics/Publications/PublicationsStatistics/
PublicationsStatisticsArticle/fs/en?CONTENT_ID $=4085951 \&$ chk $=\mathrm{HBt} 2 \mathrm{QD}$ (accessed July 2005)

5 Morgan M, Salmon R, Keppie N, Evans-Williams D, Hosein I, Looker DN. All Wales surveillance of methicillin-resistant Staphylococcus aureus (MRSA): the first year's results.J Hosp Infect 1999;41:173-9.

6 Reid J. Bloodborne MRSA infection rates to be halved by 2008. www.doh.gov.uk (accessed 1 July 2005).

7 British Society for Antimicrobial Chemotherapy, Hospital Infection Society and the Infection Control Nurses Association. Revised guidelines for the control of methicillinresistant Staphylococcus aureus infection in hospitals.J Hosp Infect 1998.39.95?-90.

8 Brown DF. Detection of methicillin/oxacillin resistance in staphylococci. J Antimicrob Chemother 2001;48(suppl 1):65-70.

9 Garner JS, Jarvis WR, Emori TG, Horan TC, Hughes JM. CDC definitions for nosocomial infections, 1988. Am J Infect Control 1988;16:128-40.

10 Lodise TP, McKinnon PS, Swiderski L, Rybak MJ. Outcomes analysis of delayed antibiotic treatment for hospital-acquired Staphylococcus aureus bacteremia. Clin Infect Dis 2003;36:1418-23.

11 Rezende NA, Blumberg HM, Metzger BS, Larsen NM, Ray SM, McGowan JE Jr. Risk factors for methicillin-resistance among patients with Staphylococcus aureus bacteremia at the time of hospital admission. Am J Med Sci 2002;323:117-23.

12 Goetz A, Posey K, Fleming J, Jacobs S, Boody L, Wagener MM, et al. Methicillin-resistant Staphylococcus aureus in the community: a hospital-based study. Infect Control Hosp Epidemiol 1999;20:689-91.

13 Lodise TP Jr, McKinnon PS, Rybak M. Prediction model to identify patients with Staphylococcus aureus bacteremia at risk for methicillin resistance. Infect Control Hosp Epidemiol 2003:24:655-61.

14 Chassagne P, Perol MB, Doucet J, Trivalle C, Menard JF, Manchon ND, et al. Is presentation of bacteremia in the elderly the same as in younger patients? Am J Med 1996;100:65-70.

15 Wyllie DH, Bowler IC, Peto TE. Bacteraemia prediction in emergency medical admissions: role of C reactive protein. J Clin Pathol 2005;58:352-6.

16 Chirouze C, Schuhmacher H, Rabaud C, Gil H, Khayat N, Estavoyer JM, et al. Low serum procalcitonin level accurately predicts the absence of bacteremia in adult patients with acute fever. Clin Infect Dis 2002;35:156-61.

17 Caterino JM, Scheatzle MD, Forbes ML, D'Antonio JA. Bacteremic elder emergency department patients: procalcitonin and white count. Acad Emerg Med 2004;11:393-6.

18 Davis KA, Stewart JJ, Crouch HK, Florez CE, Hospenthal DR. Methicillin-resistant Staphylococcus aureus (MRSA) nares colonization at hospital admission and its effect on phylococcus aureus (MRSA) nares colonization at hospital
subsequent MRSA infection. Clin Infect Dis 2004:39:776-82.

19 Huang SS, Platt R. Risk of methicillin-resistant Staphylococcus aureus infection after previous infection or colonization. Clin Infect Dis 2003;36:281-5.

20 Hori S, Sunley R, Tami A, Grundmann H. The Nottingham Staphylococcus aureus population study: prevalence of MRSA among the elderly in a university hospital. $J$ Hosp Infect 2002;50:25-9.

21 Lucet JC, Grenet K, Armand-Lefevre L, Harnal M, Bouvet E, Regnier B, et al. High prevalence of carriage of methicillin-resistant Staphylococcus aureus at hospital admission in elderly patients: implications for infection control strategies. Infect Control Hosp Epidemiol 2005;26:121-6.

22 Tacconelli E, Venkataraman L, De Girolami PC, EM DA. Methicillin-resistant Staphylococcus aureus bacteraemia diagnosed at hospital admission: distinguishing between community-acquired versus healthcare-associated strains. I Antimicrob Chemother 2004;53:474-9.

23 Morin CA, Hadler JL. Population-based incidence and characteristics of communityonset Staphylococcus aureus infections with bacteremia in 4 metropolitan Connecticut areas, 1998. J Infect Dis 2001;184:1029-34.

24 Friedman ND, Kaye KS, Stout JE, McGarry SA, Trivette SL, Briggs JP, et al. Health careassociated bloodstream infections in adults: a reason to change the accepted definition of community-acquired infections. Ann Intern Med 2002;137:791-7.

(Accepted 19 July 2005)

doi $10.1136 /$ bmj.38558.453310.8F

Nuffield Department of Clinical Laboratory Sciences, University of Oxford, John Radcliffe Hospital, Oxford OX3 9DU

David H Wyllie clinical lecturer in microbiology

Derrick Crook consultant microbiologist

Nuffield Department of Medicine, University of Oxford, John Radcliffe Hospital Tim E A Peto professor of infectious diseases

Correspondence to: D Wyllie david.wyllie@ndcls.ox.ac.uk 